\title{
Modelado De los factores ambientales Que Determinan LA DISTRIBUCIÓN DE ESPECIES SINANTRÓPICAS DE PHYSALIS
}

\author{
José Antonio López-Sandoval ${ }^{1}$, Lauro López-Mata ${ }^{1,5}$, Gustavo Cruz-Cárdenas², \\ Heike Vibrans ${ }^{1}$, Ofella Vargas ${ }^{3}$ y Mahinda Martínez ${ }^{4}$ \\ 'Postgrado en Botánica, Colegio de Postgraduados, Montecillo, Texcoco, Estado de México, México \\ ${ }^{2}$ Centro Interdisciplinario de Investigación para el Desarrollo Integral Regional (CIIDIR), \\ Instituto Politécnico Nacional-Michoacán, Jiquilpan, Michoacán, México \\ ${ }^{3}$ Universidad de Guadalajara, Centro Universitario de Ciencias Biológicas y Agropecuarias, Zapopan, Jalisco, México \\ ${ }^{4}$ Universidad de Querétaro, Facultad de Ciencias Naturales, Santiago de Querétaro, Querétaro, México. \\ ${ }^{5}$ Autor para la correspondencia. lauro@colpos.mx
}

\begin{abstract}
Resumen: El modelado de la distribución de especies sinantrópicas (malezas) y los factores ambientales que determinan dicha distribución han sido poco estudiados. Physalis tiene 90 especies distribuidas en las Américas, y algunas especies en el Viejo Mundo. México alberga cerca de 70 especies y aproximadamente 35 son endémicas. La sección Angulatae incluye diez especies, todas sinantrópicas en mayor o menor grado. Las especies se concentran en la Sierra Madre Occidental, la Sierra Madre del Sur y en la Faja Volcánica Transmexicana. El objetivo de este trabajo fue modelar e identificar las variables ambientales que determinan la distribución potencial de las diez especies de Physalis sección Angulatae. Se emplearon 524 registros revisados por especialistas en la taxonomía del grupo y 20 variables ambientales; de éstas 12 fueron climáticas, tres edáficas, dos de cobertura de la vegetación y tres de atributos topográficos. Los modelos se calcularon con el algoritmo Maxent. Los resultados del modelado mostraron que el hábitat más adecuado para la persistencia de ocho especies se definió por el índice normalizado diferencial de vegetación en los meses secos del año, la materia orgánica del suelo, la altitud y el aspecto, las cuales en conjunto explicaron entre el 73 y el $91 \%$ de la variación en su distribución. Otros factores como la precipitación total anual y la isotermalidad determinaron la distribución de $P$. crassifolia y de $P$. glabra, respectivamente. El índice normalizado diferencial de la vegetación y las propiedades de los suelos, son predictores determinantes en la distribución potencial de las especies de la sección Angulatae.
\end{abstract}

Palabras clave: máxima entropía, predictores ambientales, sección Angulatae, tomatillos.

\begin{abstract}
The modeling of the distribution of synanthropic (weedy) species and the environmental factors that determine their distribution is not well studied. Physalis has 90 species distributed in the Americas, and several in the Old World. Mexico harbors about 70 species and approximately 35 are endemic. The section Angulatae includes 10 species, all synanthropic to some degree. The species tended to concentrate in the Sierra Madre Occidental, the Sierra Madre del Sur, and the Transmexican Volcanic Belt. The aim of this work was to model and identify the environmental variables that determine the potential distribution of the ten species of Physalis sect. Angulatae. A total of 524 records that had been verified by the taxonomic experts of the group and 20 environmental variables were used; 12 were climatic, and the other eight were novel and of different types: three soil properties, two normalized differential vegetation indexes and three topographic attributes. The models were obtained with the Maxent algorithm. The results of the modelling showed that the most suitable habitat for the persistence of eight species was delimited by the normalized differential vegetation index during the dry months of the year, the soil organic matter, the elevation and the aspect, which together explained between the 73 and $91 \%$ of the variation in its distribution. Other groups of factors like total precipitation and isthermality determined the distribution of $P$. crassifolia and $P$. glabra, respectively. We show that the novel environmental factors such as the normalized differential vegetation index and the soil properties were decisive predictors in the potential distribution of the species in the section Angulatae.
\end{abstract}

Key words: maximum entropy, environmental predictors, Angulatae section, tomatillos.

$\mathbf{E}_{\mathrm{a}, \mathrm{s}}^{1}$ modelado del nicho ecológico de especies mediante algoritmos computacionales usa datos de la presencia comprobada de las especies conjuntamente con variables ambientales para producir modelos correlativos de las con- diciones más adecuadas del hábitat que determinan la distribución de las especies (Peterson et al., 2011). Las aplicaciones de estos modelos son relevantes en investigaciones sobre biología de la conservación, biogeografía, epidemiología, 
en el impacto potencial del cambio climático, en las proyecciones de ocurrencia geográfica de las especies invasoras y en la identificación de zonas y regiones geográficas que requieren ser exploradas (Guisan y Zimmermann, 2000; Hansen et al., 2001; Anderson et al., 2003; Elith y Leathwick, 2009; Peterson et al., 2011; Saupe et al., 2012). El modelado del nicho ecológico proporciona una aproximación de los factores ambientales más importantes que intervienen la distribución de las especies. Un aspecto importante que altera los patrones de distribución de ciertas especies es aquel ocasionado por las actividades humanas, las cuales suelen ocasionar que las especies estén ausentes en algunos sitios o que puedan ser desplazadas hacia ambientes menos viables para su supervivencia y persistencia.

El impacto de los disturbios antropógenos es considerable debido a que los humanos han modificado casi todas las regiones terrestres del planeta, y en consecuencia no hay garantía de que el hábitat idóneo para una especie esté accesible para ella dentro de un paisaje fuertemente fragmentado. Tanto los disturbios naturales como los antropógenos pueden tomarse en cuenta en la modelación, mediante el empleo de mapas de uso de suelo o la integración de medidas de cobertura vegetal provenientes de imágenes satelitales (Pettorelli, 2014). Las especies que tienden a distribuirse o localizarse en regiones y zonas recurrentemente perturbadas por las actividades humanas implica que éstas responden positivamente tanto a factores ambientales como a esta clase de disturbios. Así, el modelado del nicho ecológico de estas especies indica la idoneidad de su distribución potencial asociada a sitios perturbados por actividades antropógenas. Este es el caso de las plantas conocidas como malezas (arvenses y ruderales) o sinantrópicas, cuya distribución basada en la modelación de su nicho ecológico, ha sido escasamente estudiada, a pesar de su importancia para el hombre (Segura et al., 2003; Lentz et al., 2008; NúñezColín et al., 2008; García-Morales, 2011). Las plantas sinantrópicas son especies no cultivadas que prosperan en ambientes antropógenos, esto es, aquellos transformados y dominados por humanos y que están adaptadas a éstos. En una acepción amplia, especie sinantrópica es sinónimo con el término maleza (sensu Baker, 1974; Espinosa-García y Sarukhán, 1997; Villaseñor y Espinosa-García, 1998 y Espinosa-García et al., 2004).

El género Physalis (Solanaceae), con más de 75 especies a nivel mundial (Whitson y Manos, 2005), está integrado por los subgéneros: Physalis, Physalodendron, Quincula y Rydbergis. México es el centro de diversidad del subgénero Rydbergis que incluye a nueve secciones con aproximadamente 60 especies, de las cuales 35 son endémicas. En la presente investigación se estudian las diez especies que constituyen la sección Angulatae: Physalis acutifolia, P. ampla, P. angulata, P. crassifolia, P. glabra, P. lagascae, $P$. microcarpa, $P$. philadelphica, $P$. solanaceus y P. sulphurea (Martínez, 1999). Estas especies son sinantrópicas, tanto arvenses como ruderales, dos de las diez especies se cultivan y el resto representan recursos genéticos no explorados. Physalis philadelphica conocida como tomate de cáscara, es ampliamente cultivada (Serrano-Altamirano, 1998), mientras que Physalis angulata (tomate milpero) se considera en proceso de domesticación y se cultiva en algunas localidades de Jalisco (Sánchez-Martínez et al., 2008). Las poblaciones silvestres con distribución marginal de ambas especies cultivadas podrían servir como recursos genéticos valiosos para su futura experimentación y el mejoramiento genético de sus cultivos.

Las especies de la sección Angulatae se distribuyen en diversos ambientes asociados a la hetereogeneidad geológica, edáfica, tipos de vegetación y hábitats del país (Santiaguillo-Hernández et al., 2010). Por lo tanto, el entendimiento de las determinantes ambientales de la distribución espacial de especies de Physalis sección Angulatae y su modelación debe incluir además del clima, las propiedades de los suelos, el relieve del terreno, su altitud y cobertura de vegetación (índice normalizado diferencial de la vegetación, NDVI) predominantes a través de su ámbito de distribución geográfica conocida.

El objetivo de este trabajo fue modelar y predecir cuáles variables ambientales son las más importantes en la determinación de la distribución potencial de las especies de estudio. Se propone que existe un grupo limitado de factores ambientales que definen el nicho ambiental, responsable en gran medida de la distribución de las diez especies de Physalis de la sección Angulatae. Por otra parte, cada especie debe tener su propio nicho ambiental que la diferencia de las otras cogenéricas. En consecuencia se plantea responder la siguiente interrogante: ¿Qué variables ambientales definen la distribución potencial de las especies de la sección Angulatae? Adicionalmente, el conocimiento de la distribución geográfica y ecológica de las especies de la sección Angulatae en México proporcionará información útil para el trabajo de campo, para identificar zonas potenciales para su cultivo y para su conservación in situ y ex situ.

\section{Materiales y métodos}

Base de datos. La tabla 1 muestra el número de registros por especie empleados en este estudio. Se elaboró una base de datos para las diez especies del género Physalis sección Angulatae. Los 524 registros se obtuvieron de la consulta de los ejemplares de siete herbarios: Herbario Nacional de México (MEXU); Instituto Politécnico Nacional (ENCB); Colegio de Postgraduados (CHAPA); Instituto de Ecología (XAL); Universidad de Querétaro (QMEX); Universidad de Guadalajara (IBUG) e Instituto de Ecología A.C. en Pátzcuaro (IEB). Además, la base de datos se incrementó porque los especialistas del género Physalis proporcionaron los registros de sus colectas y el primer autor hizo colectas de campo adicionales. Todos los registros fueron cotejados 
Tabla 1. Especies, acrónimos, y número de registros de las diez especies de Physalis, subgénero Rydbergis, sección Angulatae empleados en el modelado de su distribución potencial.

\begin{tabular}{llc}
\hline Especie & Acrónimo & $\begin{array}{c}\text { Número } \\
\text { de registros }\end{array}$ \\
\hline Physalis philadelphica Lam. & PPHIL & 101 \\
Physalis crassifolia Benth. & PCRAS & 73 \\
Physalis solanaceus (Schltdl.) Axelius & PSOLA & 68 \\
Physalis angulata L. & PANGU & 63 \\
Physalis lagascae Roem. \& Schult. & PLAGA & 56 \\
Physalis acutifolia (Miers) Sandwith & PACUT & 53 \\
Physalis ampla Waterf. & PAMPL & 42 \\
Physalis sulphurea (Fernald) Waterf. & PSULP & 34 \\
Physalis glabra Benth. & PGLAB & 20 \\
Physalis microcarpa Urb. \& Ekman & PMICR & 14 \\
Total & & 524 \\
\hline
\end{tabular}

para tener su correcta identificación por la especialista del grupo; así como sus coordenadas geográficas.

Pruebas de aleatoriedad y análisis del patrón. Los registros por especie se sometieron a un análisis de aleatoriedad con el propósito de disminuir o eliminar la autocorrelación inherente en los registros espaciales de las especies. Cuando se presentó autocorrelación espacial en los registros, ésta se resolvió mediante la aplicación de pruebas de aleatoriedad seguidos de un análisis del patrón por especie conforme el método propuesto por Cruz-Cárdenas et al. (2014a).

Modelado de distribución. Se modeló la distribución potencial de las especies con base en los registros conocidos y su asociación con variables ambientales climáticas, edáficas, topográficas y de cobertura vegetal para todo México. El modelado de la distribución potencial empleó sólo cuatro de las capas climáticas de WorldClim (BIO1, BIO3, BIO12 y BIO15; Cruz-Cárdenas et al., 2014a). Adicionalmente, se integraron datos sobre la cobertura vegetal y topografía a partir de datos de sensores remotos, además de datos detallados de las propiedades de los suelos de México (CruzCárdenas et al., 2014b). La Tabla 2 muestra las 20 capas ambientales utilizadas en la modelación que incluyen 12 variables climáticas, tres variables de suelo, tres de atributos topográficos y dos de cobertura vegetal estacional. Las variables seleccionadas generadas para México se basaron en el trabajo de Cruz-Cárdenas et al. (2014a), el cual mostró que el uso de componentes principales como predictores ambientales genera modelos predictivos parsimoniosos, además de que las 20 variables predictivas empleadas en la modelación están libres de multicolinearidad (Cruz-Cárdenas et al., 2014a). Siguiendo las recomendaciones de CruzCárdenas et al. (2014a) de cada componente generado, se consideró sólo tomar los valores más altos de los factores de carga de las variables para el análisis de los resultados.
La configuración de Maxent fue por defecto (Phillips y Dudik, 2008), excepto por la desactivación de los módulos "Extrapolate" y "Do clamping". El formato de salida del modelo fue logístico. Para el modelado de la distribución potencial de las especies se empleó el $75 \%$ de los registros de las especies aleatoriamente distribuidas para entrenar el modelo y el $25 \%$ restantes para validarlo. El modelo se transformó a una capa booleana (presencia-ausencia). En Quantum GIS 1.7.4 (Quantum GIS Development Team, 2012) se clasificó la probabilidad de encontrar a la especie en 14 entradas. Se tomó como intervalo de probabilidad elevado para la presencia de especies del 70 al $99 \%$. De la salida de Maxent se tomó como umbral de corte el valor logístico con $10 \%$ de error de omisión (Pearson et al., 2007). El número de aciertos se obtuvo cuantificando cuantos registros de validación tienen valores logísticos por arriba del umbral de corte. Se validó el modelo con una prueba bino-

Tabla 2. Capas de los predictores ambientales empleadas en el modelado de la distribución potencial de las diez especies de Physalis. La evapotranspiración real anual (ETRA) se calculó con base en el modelo de Turc (1954): ETRA $=\mathrm{P} /\left[0.9+(\mathrm{P} / \mathrm{L})^{2}\right]^{1 / 2}$, dónde $\mathrm{P}=$ Precipitación total anual $(\mathrm{mm}), \mathrm{L}=300+25 \mathrm{~T}+0.05 \mathrm{~T}^{3}$ y $\mathrm{T}=$ Temperatura media anual $\left({ }^{\circ} \mathrm{C}\right)$.

\begin{tabular}{|c|c|}
\hline Acrónimo & Predictor ambiental \\
\hline & a) Variables climáticas \\
\hline $\mathrm{BIO} 1$ & Temperatura media anual \\
\hline $\mathrm{BIO} 3$ & Isotermalidad $(\mathrm{BIO} 1 / \mathrm{BIO} 7) \times 100$ \\
\hline $\mathrm{BIO} 4$ & Estacionalidad de la temperatura (desv. estand. $\times 100$ ) \\
\hline BIO 12 & Precipitación anual \\
\hline BIO 15 & Estacionalidad de la precipitación \\
\hline ETRA & Evapotranspiración real anual \\
\hline ETRAH & $\begin{array}{l}\text { Evapotranspiración real de la temporada lluviosa del } \\
\text { año (mayo a octubre) }\end{array}$ \\
\hline ETRAS & $\begin{array}{l}\text { Evapotranspiración real de la temporada seca del año } \\
\text { (noviembre a abril) }\end{array}$ \\
\hline $\mathrm{PPH}$ & Precipitación pluvial de la temporada lluviosa del año \\
\hline PPS & Precipitación pluvial de la temporada seca del año \\
\hline $\mathrm{TH}$ & $\begin{array}{l}\text { Temperatura promedio de la temporada lluviosa } \\
\text { del año }\end{array}$ \\
\hline \multirow[t]{2}{*}{ TS } & Temperatura promedio de la temporada seca del año \\
\hline & b) Propiedades de suelos \\
\hline CE & Conductividad eléctrica \\
\hline $\mathrm{MO}$ & Materia orgánica \\
\hline \multirow[t]{2}{*}{$\mathrm{PH}$} & Potencial de hidrogeniones \\
\hline & c) Atributos topográficos \\
\hline AS & Aspecto \\
\hline AL & Altitud \\
\hline \multirow[t]{2}{*}{ PE } & Pendiente \\
\hline & d) Índices de cobertura de la vegetación \\
\hline NDVIH & $\begin{array}{l}\text { Índice normalizado diferencial de vegetación de los } \\
\text { meses húmedos del año } 2009\end{array}$ \\
\hline NDVIS & $\begin{array}{l}\text { Índice normalizado diferencial de vegetación de los } \\
\text { meses secos del año } 2009\end{array}$ \\
\hline
\end{tabular}


mial con el software R (R Development Core Team, 2014), para evaluar si este modelo es mejor que uno aleatorio $(P$ $>0.5$ ). Además, se empleó el valor del área bajo la curva (AUC) proporcionado por la curva operada por el receptor (ROC) para evaluar la robustez de los modelos. Se acepta, en términos generales, que los valores del AUC entre $0.7 \mathrm{y}$ 0.9 para los datos de entrenamiento y de prueba del modelo, como modelos buenos y aquellos con valores mayores a 0.9 como modelos muy buenos (Peterson et al., 2011).

\section{Resultados}

Distribución conocida de las especies de la sección Angulatae. Las especies de la sección Angulatae están ampliamente distribuidas en el país, pero se concentran principalmente en la Sierra Madre Occidental, Sierra Madre del Sur y la Faja Volcánica Transmexicana (Figura 1).

La figura 2 muestra la distribución geográfica conocida de las diez especies de la sección Angulatae. Physalis ampla, $P$. lagascae y $P$. sulphurea concentran su distribución en el occidente y centro del país. Physalis crassifolia, P. glabra y $P$. acutifolia agrupan su distribución en el occidente del país, principalmente en la Península de Baja California, P. crassifolia es una especie del noroeste de México, P. glabra tiene registros en la región sur de la Península de Baja California
Tabla 3. Distribución potencial de las diez especies de la sección Angulatae y sus coincidencias con los tipos de vegetación propuestos por (Villaseñor y Ortiz, 2014).

\begin{tabular}{ll}
\hline Especies & Distribución potencial \\
\hline $\begin{array}{l}\text { Primer grupo: Physalis } \\
\text { philadelphica, P. angulata, } \\
\begin{array}{l}\text { P. lagascae, P. microcarpa, } \\
\text { P. ampla y Physalis solanaceus }\end{array}\end{array}$ & $\begin{array}{l}\text { Bosque templado, bosque tropical } \\
\text { estacionalmente seco y }\end{array}$ \\
\hline $\begin{array}{l}\text { Segundo grupo: Physalis } \\
\text { acutifolia, P. crassifolia y }\end{array}$ & Matorral xerófilo y bosque \\
P. glabra & tropical estacionalmente seco \\
\hline $\begin{array}{l}\text { P. sulphurea } \\
\end{array}$ & $\begin{array}{l}\text { Bosque tropical estacionalmente } \\
\text { seco, bosque templado, bosque } \\
\text { tropical húmedo y matorral xerófilo }\end{array}$ \\
&
\end{tabular}

y P. acutifolia a lo largo de la Planicie Costera del Pacífico, a bajas altitudes, con distribución esporádica en el sur de México. Physalis philadelphica y P. angulata se distribuyen en la mayor parte del país, excepto en la Península de Baja California; $P$. angulata se localiza principalmente en Jalisco, Tabasco y Chiapas. Physalis microcarpa presenta una distribución disyunta, en la parte norte y sur del país y Physalis solanaceus se distribuye sobre la Sierra Madre Oriental, la Faja Volcánica Transmexicana y las serranías de Oaxaca.

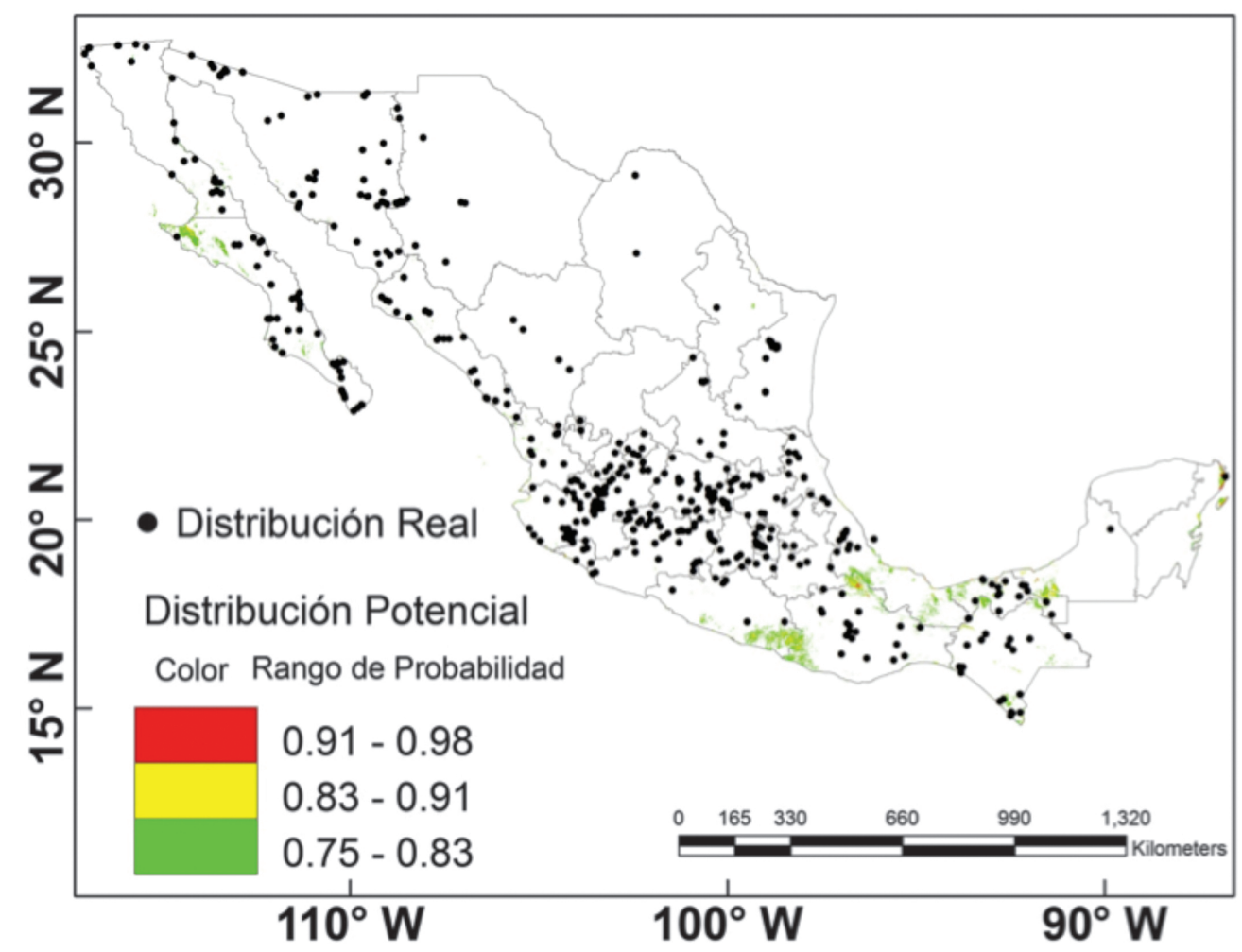

Figura 1. Distribución geográfica conocida (puntos sólidos) y distribución potencial (en color) de las especies sinantrópicas del género Physalis, subgénero Rydbergis, sección Angulatae. La distribución geográfica conocida corresponde a los registros de las diez especies de la sección Angulatae. La distribución potencial está indicada por las barras de colores con diferentes intervalos de probabilidad de ocurrencia que indican las áreas potenciales donde se pudieran encontrar las especies de la sección Angulatae. 

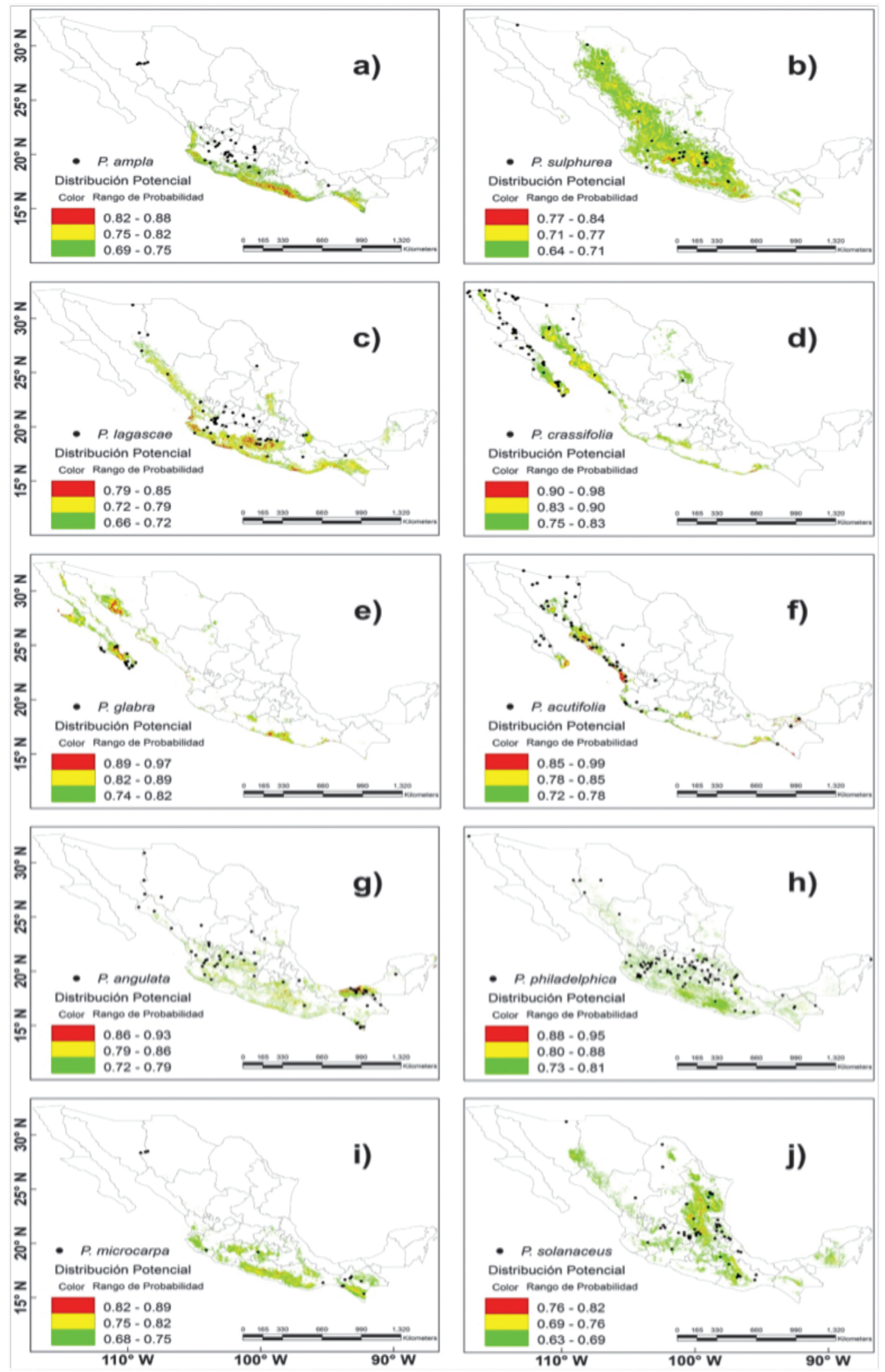

Figura 2. Distribución conocida y potencial de las diez especies sinantrópicas de Physalis sección Angulatae. El símbolo $\bullet$ indica la distribución conocida de cada especie. La distribución potencial en color indica el intervalo de idoneidad de hábitat o probabilidades de que las especies se localicen en las distintas regiones geográficas de México. 
José Antonio López-SANDOval ET AL.

Tabla 4. Porcentaje de la importancia total y relativa de los predictores ambientales particulares producidos en el modelado en Maxent. Las especies están ordenadas del valor mayor de porcentaje en el PC1. Los valores de la sección Angulatae se colocan en la primera fila.

\begin{tabular}{llcc}
\hline Especie & & $\begin{array}{c}\text { Predictores ambientales } \\
\text { (porcentaje explicado) }\end{array}$ & Total \\
\hline Sección Angulatae & PC1 (79.0) & PC7 (7.8) & PC4 (5.7) \\
Physalis ampla & PC1 (90.7) & & \\
Physalis solanaceus & PC1 (86.1) & & \\
Physalis philadelphica & PC1 (84.5) & & \\
Physalis microcarpa & PC1 (82.4) & & \\
Physalis lagascae & PC1 (81.6) & & \\
Physalis angulata & PC1 (75.2) & PC2 (8.6) & 86.1 \\
Physalis sulphurea & PC1 (73.4) & PC3 (22.6) & 84.5 \\
Physalis acutifolia & PC1 (47.8) & PC4 (33.0) & 81.6 \\
Physalis glabra & PC4 (43.9) & PC2 (30.0) & PC7 (23.1) \\
Physalis crassifolia & PC2 (37.6) & PC1 (34.2) & PC4 (23.8) \\
\hline
\end{tabular}

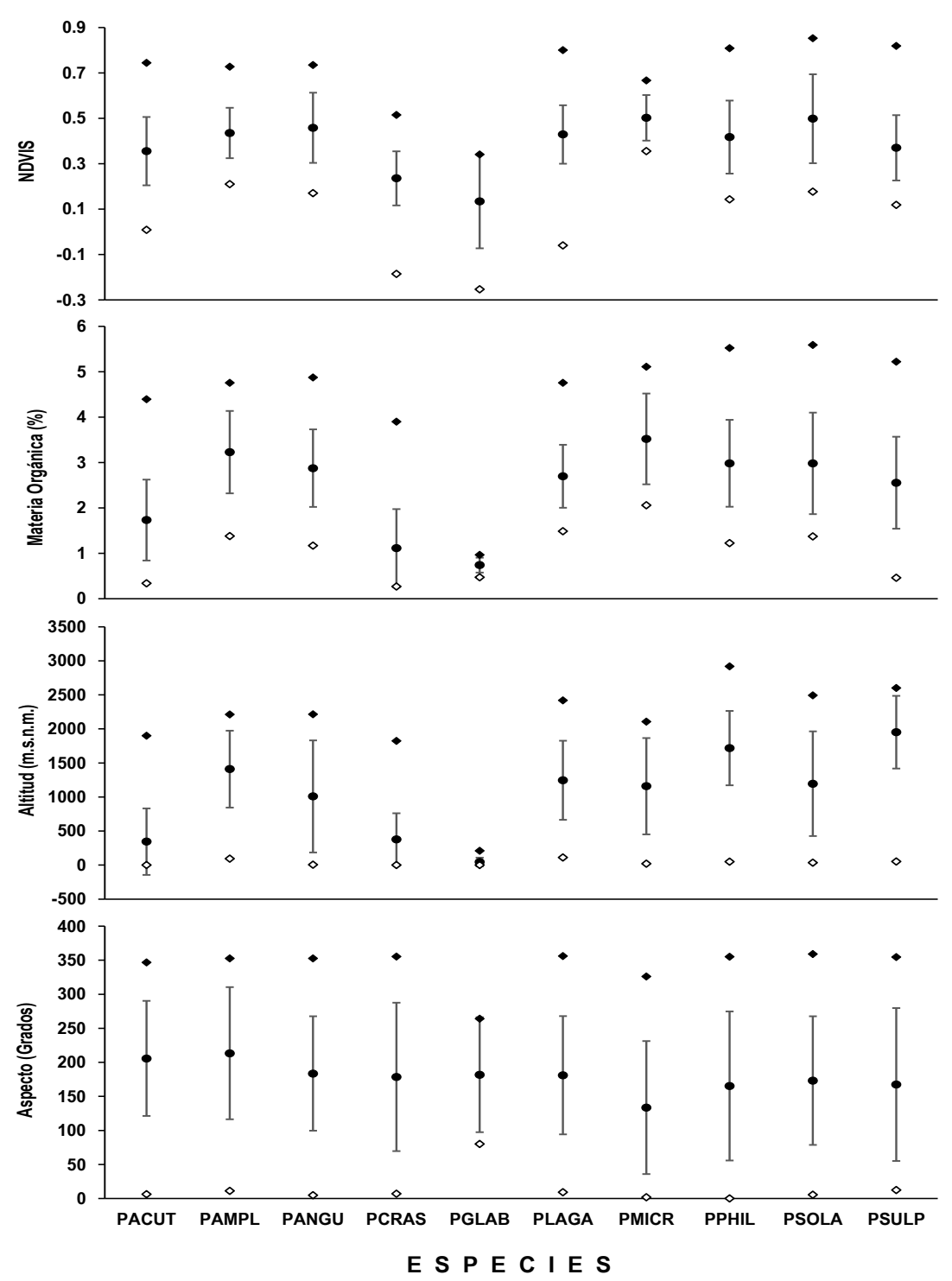

Figura 3. Estadística descriptiva de los predictores ambientales determinantes de la distribución potencial de diez especies de Physalis sección Angulatae. $\bullet=$ media, $\diamond=$ mínimo, $\diamond=$ máximo y desviación estándar representada por las barras. 
Distribución potencial de las especies de la sección Angulatae. La sección Angulatae está localizada potencialmente en bosque tropical húmedo, bosque tropical estacionalmente seco, bosque templado y matorral xerófilo. La distribución potencial de dos grupos de especies y de una especie tiene coincidencias con los tipos de vegetación de México propuestos por Villaseñor y Ortiz (2014). El primer grupo está formado por seis especies ( $P$. ampla, $P$. angulata, $P$. lagascae, $P$. microcarpa, $P$. philadelphica y $P$. solanaceus). Las tres especies del segundo grupo son $P$. acutifolia, $P$. crassifolia y $P$. glabra y únicamente $P$. sulphurea tiene diferentes coincidencias a las especies de los otros dos grupos (Tabla 3 ).

Predictores ambientales y su contribución. El análisis de los predictores ambientales arrojó siete grupos de variables o componentes principales (PC; Tabla 4), que delimitan la distribución de la sección Angulatae y de las especies en particular y la proporción de la varianza que explica cada uno de ellos. El grupo PC1 explicó una elevada proporción de la varianza (79\%) registrada en los datos de toda la sección Angulatae y proporciones de más de $73 \%$ para siete de las 10 especies, y sólo el $48 \%$ para una de las tres restantes. Para dos especies resultaron más importantes otros dos grupos de predictores, el PC2 para Physalis crassifolia y el PC4 para P. glabra. Los factores de carga más importantes de las variables en el PC1 fueron: el NDVI de los meses secos del año, la materia orgánica del suelo, el modelo digital de elevación (altitud) y el aspecto del terreno. En cambio, los factores más altos del PC2 que explican la distribución potencial de $P$. crassifolia fueron: la precipitación total anual; en cambio, aquellos del PC4 que explican la distribución de $P$. glabra fueron: la isotermalidad y la precipitación pluvial durante los meses secos del año.

Perfiles bioclimáticos de las especies de Physalis sección Angulatae. Los valores del NDVIS fluctuaron entre $0.35 \mathrm{y}$ 0.5 lo que sugiere que todas las especies prefieren ambientes que aún en invierno conservan algo de humedad (Figura $3)$. En cuanto a la materia orgánica siete especies prefieren suelos que varían de medianamente ricos a ricos (2.41 a 4.2 $\%)$. Sólo $P$. acutifolia puede desarrollarse en suelos medianamente pobres (1.21 a $1.8 \%$; Figura 3) según la escala de materia orgánica de Velasco-Molina 1983). Siete especies se distribuyen a altitudes intermedias y únicamente $P$. acutifolia se encuentra en bajas altitudes (Figura 3). Con respecto a los cambios topográficos y orientación de la ladera (aspecto), los promedios de siete especies muestran una preferencia para una exposición sur-suroeste y $P$. microcarpa prefiere exposiciones sureste (Figura 3 ).

La prueba binomial aplicada a las diez especies de la sección Angulatae para la validación del modelo, obtuvo valores por arriba del umbral de corte $(P>0.05)$. La precisión del modelo generada para Physalis ampla, $P$. microcarpa, $P$. solanacea y $P$. sulphurea fueron estadísticamente similares
Tabla 5. Áreas bajo la curva (AUC) operada por el receptor (ROC), para los datos de entrenamiento y los de prueba de los modelos resultantes. Las especies están ordenadas por orden alfabético. Los valores de la sección Angulatae se colocan en la primera fila.

\begin{tabular}{lcc}
\hline Especie & Entrenamiento & Prueba \\
\hline Sección Angulatae & 0.929 & 0.842 \\
Physalis acutifolia & 0.947 & 0.958 \\
Physalis ampla & 0.940 & 0.907 \\
Physalis angulata & 0.950 & 0.936 \\
Physalis crassifolia & 0.925 & 0.892 \\
Physalis glabra & 0.893 & 0.904 \\
Physalis lagascae & 0.920 & 0.942 \\
Physalis philadelphica & 0.892 & 0.927 \\
Physalis microcarpa & 0.958 & 0.929 \\
Physalis solanaceus & 0.949 & 0.940 \\
Physalis sulphurea & 0.938 & 0.951 \\
\hline
\end{tabular}

porque incluyen el $100 \%$ de sucesos con registros de validación. Para P. philadelphica (98\%), P. acutifolia (97\%), P. crassifolia (95\%), P. angulata (93\%) y P. lagascae (90\%) no fueron estadísticamente diferentes; la precisión de estas cinco especies fue más alta que P. glabra $(86 \%)$ y del conjunto de la sección Angulatae (76 \%).

La Tabla 5 muestra el área bajo la curva (AUC) operada por el receptor (ROC), tanto para los resultados del entrenamiento como para los de prueba para la sección Angulatae y para cada una de las especies estudiadas. Es notorio que en todos los casos, excepto dos, el área bajo la curva ROC resulta mayor de 0.9 , lo que indica que los modelos resultantes son modelos muy buenos y que los dos restantes son modelos buenos (Peterson et al., 2011).

\section{Discusión}

Distribución conocida de las especies de Physalis sección Angulatae. El presente trabajo permitió delimitar el hábitat de las especies de la sección Angulatae a altitudes desde el nivel del mar hasta los 2,500 $\mathrm{m}$, pero principalmente a altitudes intermedias (2,000 a 2,500 m). Solo P. philadelphi$c a$ se distribuye a altitudes mayores $(2,700 \mathrm{~m})$. La sección Angulatae prospera principalmente en climas cálidos subhúmedos propios del bosque tropical estacionalmente seco, pero también en el bosque templado con climas templados subhúmedos y lluvias en verano y algunas especies en climas secos. Las especies de esta sección tienden a concentrarse en la Sierra Madre Occidental y del Sur, así como la Faja Volcánica Transmexicana. El modelado sugirió que la región que requiere incrementar las colectas es la Sierra Madre Occidental, especialmente las partes altas de Sonora, Chihuahua, Sinaloa y Durango.

Distribución potencial de las especies de Physalis sección Angulatae. Las tres especies de Physalis con distribución 
potencial amplia ( $P$. lagascae, $P$. solanaceus y $P$. sulphurea) se desarrollan en los ambientes del bosque tropical estacionalmente seco y del matorral xerófilo. Santiaguillo-Hernández et al. (2010) registró preferencia de estas tres especies por suelos tipo Feozem. Este reporte se apoya con nuestros resultados acerca de la importancia de la materia orgánica, abundante en suelos tipo Feozem, para la distribución en la sección y para estas especies.

Con base en los resultados, ocho especies de Physalis de la sección Angulatae se agruparon por el tipo de vegetación en que habitan. Por ejemplo, P. ampla, P. acutifolia, $P$. angulata, $P$. lagascae, $P$. microcarpa, $P$. philadelphica, $P$. solanacea y $P$. sulphurea se distribuyen tanto en el bosque templado como en el bosque tropical estacionalmente seco. Su nicho ambiental es amplio y podría explicarse por el carácter sinantrópico de estas especies, es decir por el elevado potencial de dispersión y movilidad antropo-dependiente. Physalis philadelphica, la especie de mayor sinantropía y la más cultivada, se desarrolla en la más amplia gama de tipos de vegetación; su distribución es similar a la de Solanum stoloniferum, una especie también semidomesticada (Luna-Cavazos et al., 2012). Otras dos especies, $P$. crassifolia y P. glabra, prosperan en los climas secos del matorral xerófilo y del bosque tropical estacionalmente seco. Su nicho ecológico es más restringido, sus áreas de distribución son más pequeñas y también son las menos sinantrópicas.

Physalis philadelphica y $P$. angulata tienen algunas características bioclimáticas especiales. Por ejemplo, $P$. philadelphica es una de las especies que se distribuye a mayor altitud y tolera las más bajas temperaturas promedio de la temporada lluviosa del año. Estas capacidades le permiten desarrollarse en gran parte del territorio mexicano y posiblemente por ello es la especie que se cultiva más ampliamente. Physalis angulata se distribuye en los intervalos más amplios de altitud y bajo las condiciones más elevadas de evapotranspiración real anual. En Jalisco coinciden las áreas de cultivo de ambas especies (Santiaguillo-Hernández et al., 2010). Otras áreas que parecen idóneas para el cultivo de $P$. angulata se localizan en Michoacán, Guerrero y Tabasco, en lugares donde la temperatura media fluctúa entre 20 y $22{ }^{\circ} \mathrm{C}$, intervalo de temperatura que requiere el cultivo de $P$. philadelphica (Serrano-Altamirano, 1998).

¿Existe un patrón de factores ambientales que determina la distribución potencial de la sección Angulatae? Los resultados de este estudio, soportan la hipótesis de que existe un patrón de factores que delimitan la distribución de ocho de las diez especies de la sección Angulatae, es decir, ocho especies explicaron su distribución por las mismas variables. Tales variables incluyeron un espectro de factores ambientales pertinentes y no sólo las variables climáticas que son el estándar en el modelado de la distribución de especies. Se muestra que las capas ambientales no convencionales incluidas en este trabajo, resultaron importantes y determinaron parcialmente estos patrones.

Para Martinuzzi et al. (2008), los valores del NDVIS durante la temporada seca del año varían de bajos a intermedios (0.35-0.5) y sugieren la presencia de matorral y bosque abierto. Para las ocho especies de la sección Angulatae el NDVIS indica, si bien, éstas crecen en la temporada húmeda, prosperan principalmente en ambientes con cobertura vegetal que varía de baja a intermedia, aún en la estación seca del año. Esto coincide con las observaciones de campo y con los registros en las etiquetas de herbario y el hecho de que a menudo crecen en hábitats sinantrópicos. Otras dos especies (Physalis crassifolia y P. glabra) para este mismo factor tienen comportamiento diferente.

Está bien documentado que la materia orgánica del suelo mejora sus propiedades físicas, químicas y biológicas y que su cantidad depende de factores como la incorporación de restos orgánicos, la textura del suelo, la aireación, la humedad y los factores climáticos (Julca-Otiniano et al., 2006). De todas las especies estudiadas, únicamente Physalis acutifolia, crece en suelos pobres en materia orgánica. El único reporte conocido de respuesta similar es para Physalis puberula Fernald una especie de la sección Coztomatae del género Rydbergis la cual también se desarrolla en suelos pobres en materia orgánica (Mera-Ovando, 1987).

La importancia de la altitud, radica en que ésta determina en gran medida los parámetros de casi todos los elementos climáticos como la temperatura, precipitaciones y radiación solar. Todas las especies de la sección Angulatae se distribuyen en altitudes intermedias, con excepción de $P$. acutifolia que se localiza en bajas altitudes (Santiaguillo-Hernández et al., 2010) y de $P$. angulata y $P$. lagascae que también se reportan desde el nivel del mar (Vargas-Ponce et al., 2003). Por otra parte, el aspecto influye en la incidencia de luz y en las características del suelo y este en la distribución geográfica de las especies, por lo que su inclusión y análisis en el patrón de distribución de las especies resultó pertinente. El aspecto, por sí mismo, es una variable ambiental relevante en cualquier sistema montañoso y en este contexto, las especies de Physalis estudiadas presentaron preferencias por laderas con orientación sur.

Estudios recientes han mostrado la importancia de incluir variables no climáticas para mejorar el valor predictivo de los modelos. Por ejemplo, Dubuis et al. (2013) modelaron la distribución de 115 especies en Suiza, con solo datos climáticos, y con una amplia gama de otros factores. Su trabajo mostró que los factores mejoraron considerablemente las predicciones del modelado, destacándose el valor de $\mathrm{pH}$ y el nitrógeno del suelo. Coudun et al. (2006) modelaron la distribución de Acer campestre y encontraron que el valor de $\mathrm{pH}$ tenía una influencia más importante que la de los factores climáticos. Llama la atención que en este trabajo el valor de $\mathrm{pH}$ resultó ser un factor ambiental poco relevante para la distribución de las especies de Physalis, a pesar de 
que en un estudio de especies arvenses de la región de Puebla y Tlaxcala fue determinante para la formación de comunidades (Vibrans, 1998). Debe aclararse que las especies de la sección Angulatae generalmente ocurren sobre suelos que varían de neutros a alcalinos; mientras que en los trabajos de Dubuis et al. (2013) y de Coudun et al. (2006) el pH contribuyó a explicar la distribución de especies acidófilas. Por otra parte, la heterogeneidad ambiental climática en nuestra región de estudio es considerablemente mayor que en la de aquellos estudios.

La precipitación total anual es el factor climático más importante que explica la distribución de $P$. crassifolia. Para $P$. glabra los factores más importantes en su distribución por orden de importancia son la isotermalidad y la precipitación pluvial durante los meses secos del año. La distribución de la lluvia a lo largo del año en México es de suma importancia para la vida vegetal y su distribución geográfica en la República Mexicana está íntimamente ligada, entre otros factores, con la orografía y con la circulación del aíre atmosférico; aproximadamente $70 \%$ de la precipitación pluvial se registra durante los meses de mayo a octubre (García, 2003). En México, la isotermalidad, tiene una gran variación térmica y está influenciada principalmente por la altitud.

\section{Conclusiones}

Este trabajo muestra que la gama de factores ambientales empleados influencian determinantemente en la distribución de las diez especies de la sección Angulatae estudiadas. Se coincide con otros trabajos recientes que sugieren que una ampliación pertinente de los predictores ambientales mejora el valor predictivo de su modelado y enriquece la información sobre la ecología de las especies (Williams et al., 2012).

Los datos analizados indican que las especies de Physalis sección Angulatae están distribuidas en todo México, pero concentradas mayoritariamente en la Sierra Madre Occidental, la Sierra Madre del Sur y la Faja Volcánica Transmexicana. Habitan principalmente las regiones subhúmedas - tropicales y templadas - hasta áridas, pero con cierta humedad durante los meses secos del año y son escasas en la vegetación muy húmeda. El análisis identificó al grupo de variables que definen el nicho ambiental de ocho especies de la sección Angulatae; mientras que otros predictores definen al nicho ambiental de las otras dos especies.

Finalmente y a partir de los resultados mostrados en este trabajo, se propone que en la modelación de especies de plantas se deben incluir integralmente variables de clima, de relieve del terreno, características del suelo (no solo tipos) y de cobertura vegetal. Otros predictores ecológicos y biológicos, como son la radiación solar, las interacciones biológicas y la influencia humana directa, pueden ser también de gran interés en el modelado de la distribución de especies de plantas.

\section{Agradecimientos}

El autor principal agradece al Consejo Nacional de Ciencia y Tecnología por la beca otorgada (convenio No. 85447) para llevar a cabo sus estudios de doctorado. Los atinados comentarios y sugerencias de los revisores mejoraron el manuscrito sustancialmente.

\section{Literatura citada}

Anderson R.P., Lew D. y Peterson A.T. 2003. Evaluating predictive models of species distributions: criteria for selecting optimal models. Ecological Modelling 162:211-232.

Baker H.G. 1974. The evolution of weeds. Annual Review of Ecology and Systematics 5:1-24.

Coudun C., Gégout J.C., Piedallu C. y Rameau J.C. 2006. Soil nutritional factors improve models of plant species distribution: an illustration with Acer campestre (L.) in France. 2006. Journal of Biogeography 33:1750-1763.

Cruz-Cárdenas G., López-Mata L., Villaseñor J.L. y Ortiz E. 2014a. Potential species distribution modeling and the use of principal component analysis as predictor variables. Revista Mexicana de Biodiversidad 85: 189-199.

Cruz-Cárdenas G., López-Mata L., Ortiz-Solorio C.A., Villaseñor J.L., Ortiz E., Silva J.T. y Estrada-Godoy F. 2014b. Interpolation of Mexican soil properties at a scale of 1:1,000,000. Geoderma 213:29-35.

Dubuis A., Giovanettina S., Pellissier L., Pottier J., Vittoz P. y Guisan A. 2013. Improving the prediction of plant species distribution and community composition by adding edaphic to topo-climatic variables. Journal of Vegetation Science 24:593-606.

Elith J. y Leathwick J.R. 2009. Species distribution models: ecological explanation and prediction across space and time. Annual Review of Ecology, Evolution and Systematics 40:677-697.

Espinosa-García F.J., Villaseñor J.L. y Vibrans H. 2004. Geographical patterns in native and exotic weeds of Mexico. Weed Technology 18:1552-1558.

Espinosa-García F.J. y Sarukhán J. 1997. Manual de Malezas del Valle de México, Claves, Descripciones e Ilustraciones. Universidad Nacional Autónoma de México/Fondo de Cultura Económica, México D.F.

García E. 2003. Distribución de la precipitación en la República Mexicana. Investigaciones Geográficas, Boletín del Instituto de Geografía. UNAM 50:67-76.

García-Morales M.E. 2011. Relaciones filogenéticas con enfoque hacia la conservación de las Spondiadeae en América. Tesis maestría, Universidad Autónoma del Estado de México, Toluca. 167 pp.

Guisan A. y Zimmermann N.E. 2000. Predictive habitat distribution models in ecology. Ecological Modelling 135:147-186.

Hansen A.J., Neilson R.P., Dale H.V., Flather C.H., Iverson L.R., Currie D.J., Shafer S., Cook R. y Bartlein P.J. 2001. Global change in forest: Responses of species, communities, and biomes. BioScience 51:765-779.

Julca-Otiniano A., Meneses-Florián L., Blas-Sevillano R. y BelloAmez S. 2006. La materia orgánica, importancia y experiencias de uso en la agricultura. IDESIA 24:49-61.

Lentz D.L., Bye R. y Sánchez-Cordero V. 2008. Ecological niche modeling and distribution of wild sunflower (Helianthus an- 
nuus L.) in Mexico. International Journal of Plant Sciences 169:541-549.

Luna-Cavazos M., Romero-Manzanares A. y García-Moya E. 2012. Distribución geográfica de Solanum tuberosos silvestres de México y su relación con factores del medio. Interciencia 37:355-362.

Martínez M. 1999. Infrageneric taxonomy of Physalis. En: Nee M.D., Symon E.R., Lester N. y Jessop J. P. Eds. Solanaceae IV: Advances in Biology and Utilization, pp. 275-283, The Royal Botanical Garden, Kew, Londres.

Martinuzzi S., Gould W.A., Ramos-Gonzalez O.M., Martínez-Robles A., Calle-Maldonado P., Pérez-Buitrago N. y Fumero-Caban J.J. 2008. Mapping tropical dry forest habitats integrating Landsat NDVI, Ikonos imagery, and topographic information in the Caribbean Island of Mona. Revista de Biología Tropical 56:625-639.

Mera-Ovando L.M. 1987. Estudio comparativo del proceso de cultivo de la arvense Physalis chenopodifolia Lamark y Physalis philadelphica var. philadelphica cultivar Rendidora. Tesis de maestría, Colegio de Postgraduados, Montecillo, Texcoco. $112 \mathrm{pp}$.

Núñez-Colín C.A., Nieto-Ángel R., Barrientos-Priego A.F., Segura S., Sahagún-Castellanos J. y González-Andrés F. 2008. Distribución y caracterización eco-climática del género Crataegus L. (Rosaceae, Subfam. Maloideae) en México. Revista Chapingo Serie Horticultura 14:177-184.

Pearson R.G., Raxworthy C.J., Nakamura M. y Peterson A.T. 2007. Predicting species distribution from small numbers of occurrence records: a test case using cryptic geckos in Madagascar. Journal of Biogeography 34:102-117.

Peterson A.T., Soberón J., Pearson R.G., Anderson R.P., MartínezMeyer E., Nakamura M. y Araújo M.B. 2011. Ecological Niches and Geographic Distributions. Princeton University Press, Princeton, Nueva Jersey.

Pettorelli N. 2014. The Normalized Difference Vegetation Index. Oxford University Press, Oxford.

Phillips S.J. y Dudik M. 2008. Modeling of species distributions with Maxent: new extensions and a comprehensive evaluation. Ecography 31:161-175.

Quantum GIS Development Team. 2012. Quantum GIS Geographic Information System. Open Source Geospatial Foundation Project. <www.qgis.osgeo.org>

R Development Core Team. 2014. R: A language and environment for statistical computing. R Foundation for Statistical Computing, Vienna, Austria.

Santiaguillo-Hernández J.F., Cedillo-Portugal E. y Cuevas-Sánchez J.A. 2010. Distribución Geográfica de Physalis spp. en México.
Universidad Autónoma Chapingo, Chapingo, Texcoco, Estado de México. Prometeo Editores S.A de C.V, Guadalajara.

Sánchez-Martínez J., Vargas-Ponce O. y Zamora-Tavares P. 2008. Cultivo tradicional de Physalis angulata L. (Solanaceae) una especie de tomatillo silvestre de México. Avances en la investigación científica. XIX Semana Nacional de investigación científica. Centro Universitario de Ciencias Biológico y Agropecuarias, Guadalajara.

Saupe E.E., Barve V., Myers C.E., Soberón J., Barve N., Hensz C.M., Peterson A.T., Owens H.L. y Lira-Noriega A. 2012. Variation in niche and distribution model performance: The need for a priori assessment of key causal factors. Ecological Modelling 237-238:11-22.

Segura S., Coppens G., López L., Grum M. y Guarino L. 2003. Mapping the potential distribution of five species of Passiflora in Andean countries. Genetic Resources and Crop Evolution 50: 555-566.

Serrano-Altamirano A.D. 1998. Determinación del intervalo óptimo de cosecha y descripción fenológica de tomate de cáscara (Physalis ixocarpa Brot.) tipo salamanca. Tesis licenciatura, Universidad Autónoma Chapingo, Chapingo, 54 pp.

Turc L., 1954. Le bilan d'eau des sols: relation entre les précipitations, l'évaporation et l'écoulement. Annales Agronomiques 5:491-595.

Vargas-Ponce O., Martínez y Díaz M. y Dávila-Aranda P.A. 2003. La familia Solanaceae en Jalisco. El género Physalis. Universidad de Guadalajara, Guadalajara.

Velasco-Molina H.A. 1983. Problemario. Uso y Manejo del Suelo. Teoría y Laboratorio. Limusa, México, D.F.

Vibrans H. 1998. Urban weeds of Mexico City. Floristic composition and important families. Anales del Instituto de Biología de la Universidad Nacional Autónoma de México. Serie Botánica 69:37-69.

Villaseñor J.L. y Espinosa-García F.J. 1998. Catálogo de Malezas de México. Universidad Nacional Autónoma de México/ Consejo Nacional Consultivo Fitosanitario/ Fondo de Cultura Económica, México, D.F.

Villaseñor J.L. y Ortiz E. 2014. Biodiversidad de las plantas con flores (División Magnoliophyta) en México. Revista Mexicana de Biodiversidad Supl. 85:S134-S142.

Whitson M. y Manos P.S. 2005. Untangling Physalis (Solanaceae) from the Physaloids: A two-gene Phylogeny of the Physalinae. Systematic Botany 30:216-230.

Williams K.J., Belbin L., Austin M.P., Stein J.L. y Ferrier S. 2012. Which environmental variables should I use in my biodiversity model? International Journal of Geographical Information Science 26:2009-2047.

Recibido: 6 de julio de 2014

Aceptado: 1 de diciembre de 2014 